\title{
¿En España es diferente...? Mujeres inmigrantes dominicanas y marroquíes
}

\section{Carmen Gregorio Gil}

Universidad de Granada. Departamento de Antropología y Trabajo Social

Escuela Universitaria de Trabajo Social

C/ Rector López Argüeta s/n. 18071 Granada. Spain

hiperico@correo.cop.es

\section{Ángeles Ramírez Fernández}

Universidad Autónoma de Madrid. Taller de Estudios internacionales Mediterráneos (TEIM)

Departamento de Estudios Internacionales Mediterráneos

Campus de Cantoblanco. 28049 Madrid. Spain

angeles.ramirez@uam.es

\section{Resumen}

En los últimos años, se asiste a un importante incremento de la inmigración femenina en España, sobre todo en las grandes ciudades. Las autoras comparan dos colectivos de mujeres inmigrantes, el marroquí y el dominicano, a partir de su inserción en el mercado de trabajo, concretamente en el sector del servicio doméstico y la prostitución. Los cambios que lleva consigo esta inserción son analizados a lo largo del artículo. El lugar que estas mujeres ocupan en los países de origen dentro del ámbito doméstico va a ser determinante en la autopercepción y consecuencias de estos cambios.

Palabras clave: inmigración femenina, España, República Dominicana, Marruecos, servicio doméstico, prostitución, valor del trabajo.

\section{Abstract. ¿Is it different in Spain...? Dominican and Moroccan Immigrant Women}

In the last years, female migration has increased in Spain, in big cities above all. The authors compare two female migrant groups: Moroccan and Dominican, taking as starting point their insertion in the labour market, specifically Domestic Service and Prostitution. The changes caused by this integration are analysed in this article. The understanding of the situation in the household of origin is essential to explain the self-perception of these changes and their consequences.

Key words: female immigration, Spain, Dominican Republic, Morocco, domestic service, prostitution, value of work.

\section{Sumario}

\section{Introducción}

2. El valor social del trabajo de la mujer en la República Dominicana y en Marruecos
3. Mujeres dominicanas y marroquíes: diferencias en la percepción de su emigración laboral

4. Conclusiones

Bibliografía 


\section{Introducción}

Cuando acometimos la tarea de llevar a cabo un análisis comparativo de dos grupos diferenciados por su origen nacional -Marruecos y República Dominicana-, se presentaron dos dificultades.

La primera dificultad tenía que ver con el intento de evitar un cierto esencialismo cultural, en el que en ocasiones se cae al hacer comparaciones entre grupos diferenciados por su origen nacional. Tanto desde la perspectiva del estudio de la migración tomando como marco conceptual la interacción entre los sistemas de género y los procesos migratorios en el origen de las migraciones y en los cambios consecuentes ${ }^{1}$, como desde el estudio del islam en cuanto ideología de género y los cambios con la emigración al extranjero ${ }^{2}$, nos parecía necesario abordar esta comparación a partir de una serie de presupuestos teóricos que conjuraran el peligro esencialista al que antes se ha aludido. Dichos presupuestos se relacionan con la posición de género que tienen las mujeres, tanto en sus sociedades de origen como en las sociedades a donde emigran, posición que condiciona la percepción de los cambios que la emigración provoca en ellas.

La segunda dificultad tenía relación con la delimitación de los aspectos concretos del cambio que iban a ser comparados. La búsqueda de aspectos comparables venía condicionada desde el principio por las diferencias existentes en cada uno de nuestros trabajos de investigación. Si bien en las dos investigaciones se estudiaban aspectos relacionados con el cambio, cuestiones como la perspectiva de análisis, la metodología y los datos obtenidos por cada una de nosotras eran substancialmente diferentes. Por tanto, la elección de los aspectos que íbamos a comparar debía responder, en primer lugar, a la posibilidad real de tener datos comparables con un mismo nivel de profundidad. En segundo lugar, las cuestiones que se iban a comparar deberían ser significativas en la vida de las mujeres en su nueva realidad como inmigrantes. Entonces encontramos un aspecto que cumplía las dos condiciones: había sido estudiado en profundidad en ambos trabajos, lo que suponía que disponíamos de un importante acervo de datos, y además constituía una cuestión fundamental en la vida de las mujeres inmigrantes. Nos referimos al empleo de estas mujeres en España. Decidimos, entonces, realizar el análisis del cambio que la inmigración suponía para las mujeres inmigrantes dominicanas y marroquíes estudiadas a partir de algo que compartían todas ellas: su inserción en sectores marginales de un limitado mercado de trabajo, como son el servicio doméstico y la prostitución. Esta inserción se explica por una situación similar que tiene que ver con su posición de género, clase social, estereotipos culturales y extranjería ${ }^{3}$. Sin embargo, lo que nos interesaba no era tanto adentrarnos en el contexto de esta situación cuanto

1. Perspectiva adoptada en el trabajo de Gregorio (1996a, 1998a).

2. Ramírez (1998).

3. Análisis que ha sido realizado en otro lugar (Gregorio, 1996a, 1998b). 
indagar en las diferentes interpretaciones que las mujeres estaban haciendo de su situación.

Los datos que se van a utilizar en este artículo están basados en los respectivos trabajos de campo de las autoras, tanto en la sociedad de origen de las mujeres inmigrantes marroquíes y dominicanas como en la sociedad de llegada, en concreto la ciudad de Madrid. El contexto de origen seleccionado de la emigración dominicana ha sido la región suroeste del país. Dicha región se ha constituido, desde finales de los años ochenta, en un verdadero epicentro expulsor de población inmigrante a España y en concreto a la Comunidad de Madrid, siendo en su mayoría mujeres. La región se caracteriza con respecto a otras regiones de la República Dominicana por su ruralidad y por ser una de las más pobres. Las actividades económicas predominantes en la región son las agropecuarias - en muchos casos de subsistencia- y las actividades comerciales, sobre todo las de carácter informal. Respecto a las zonas de emigración en Marruecos, y dado que la mayoría de las mujeres trabajadoras vienen de las áreas urbanas, el trabajo de campo se centró en dos ciudades, Salé y Tánger. En concreto, las zonas escogidas fueron dos barrios, antes periféricos, hoy integrados en dichas ciudades. Estos barrios son lugar de viejo asentamiento de otros inmigrantes marroquíes, que proceden del interior del país. Podría hablarse de ellos como de zonas populares, pero no marginales.

\section{El valor social del trabajo de la mujer en la República Dominicana y en Marruecos}

Como en la mayor parte de las sociedades, en la República Dominicana las mujeres son definidas fundamentalmente a partir de su función social como madres y esposas. El trabajo está claramente dividido según género. Las mujeres deben encargarse del trabajo de reproducción dentro de sus hogares ${ }^{4}$, mientras que los hombres deben ser los que busquen el sostén económico a la familia fuera del hogar. Esta división del trabajo se muestra muy persistente al sustentarse en creencias sobre la diferencia biológica entre hombres y mujeres. Como señalan Pou y otros (1987) en su trabajo sobre la mujer rural en la República Dominicana, existe la creencia de que «los hombres son más fuertes por naturaleza y por ende, pueden realizar los trabajos más duros, por lo que a ellas les corresponden los trabajos más débiles» (1987: 202-203). El papel de la mujer como productora y generadora de ingresos está infravalorado, y esto se debe, fundamentalmente, a dos consideraciones de tipo ideológico. La primera, a que el trabajo que realizan las mujeres dentro del hogar, aunque sea productivo, se considera de naturaleza femenina y, por tanto, débil y de poco valor. La segunda, a que el trabajo que las mujeres desempeñan fuera del grupo doméstico, aunque genere un ingreso monetario, se considera como una ayuda.

4. Donde se incluyen todas las actividades que se dirigen a la reproducción del hogar, tengan o no valor de cambio. 
A las ideologías de género se añaden las de parentesco, que definen todo el desempeño de la mujer, madre y esposa, en el marco de su relación afectiva de carácter altruista con los diferentes miembros del grupo doméstico. La aportación económica que hacen las mujeres a sus hogares no es reconocida, ni por ella misma, ni por su sociedad, como consecuencia de esta ideología. Por el contrario, la aportación monetaria del hombre se sobrevalora y constituye una fuente de prestigio.

Las consideraciones ideológicas con respecto a la división sexual del trabajo distorsionan el valor productivo real que hombres y mujeres tienen dentro de sus grupos domésticos. Algunos estudios realizados en la región suroeste de la República Dominicana ${ }^{5}$ constatan que sin la aportación económica de la mujer se acentúan las condiciones de indigencia, y se ve dificultada la reproducción del hogar rural. Incluso en hogares donde el ingreso monetario de la mujer es menor al ingreso del hombre, su papel para garantizar la reproducción del hogar es mucho más importante que el de éste, bien porque la totalidad de sus ingresos se utilizan en la reproducción del grupo doméstico, bien porque, en mayor medida que el hombre, las mujeres producen dentro del hogar bienes que tienen un valor de cambio.

Las estrategias económicas puestas en marcha dentro de los hogares dominicanos vienen determinadas por las ideologías de género. Así, se prioriza que el hombre sea el que traiga el sustento al hogar, trabajando fuera de éste, y que la mujer se quede en el hogar encargándose de las tareas reproductivas. Sin embargo, ante la crisis económica que experimentan los hogares, las prescripciones ideológicas no se han hecho realidad dentro de los hogares más pobres. Como consecuencia de esto, las mujeres y los hombres participan indistintamente de las estrategias económicas que tienen lugar fuera del hogar: la asalarización de una parte o de todos los miembros del grupo doméstico sea dentro del mercado local o nacional, la realización de actividades comerciales por cuenta propia, la utilización de diferentes actividades informales dentro del sector servicios y la emigración internacional.

Las mujeres dominicanas se han incorporado al mercado laboral de forma significativa en las últimas décadas. A pesar de esta incorporación, el panorama no es muy alentador, puesto que sigue operando la desigualdad sexual en el mercado de trabajo que las limita a ocupar sobre todo empleos feminizados, con bajos salarios y en las escalas jerárquicas inferiores. Diferentes estudios han señalado la discriminación, tanto horizontal como vertical, que padecen las mujeres dominicanas dentro del mercado de trabajo (Báez, 1987; CELADE, 1988, Valdés y Gomariz, 1993). La mayoría de las mujeres en la República Dominicana se encuentran, o bien desempeñando puestos de trabajo dentro de los sectores típicamente femeninos — discriminación horizontal—, o bien ocupando los puestos de trabajo más bajos de la escala salarial y con menor capacidad de decisión. Según datos elaborados por Valdés y Gomariz (1993) para 
1991, las mujeres se concentran de forma mayoritaria en el sector servicios — cerca del 80\%—, estando los hombres distribuidos más equitativamente en los tres sectores - $37,6 \%$ en la agricultura, $16 \%$ en la industria y $46,4 \%$ en los servicios-. Según datos de la encuesta ENDESA-1991, dentro del sector servicios la mayor parte de las mujeres están concentradas en los servicios de hogar - suponen el $28 \%$ del total de la población ocupada-, y en las actividades relacionadas con el comercio - un $24,4 \%$ del total de la población ocupa$\mathrm{da}$ - Esto se debe al crecimiento de las ciudades y del sector terciario de la economía, fruto del modelo de desarrollo exportador de servicios que atrae la mano de obra femenina ${ }^{6}$. Así, por ejemplo, las mujeres han sido la mano de obra favorita de las industrias de capital extranjero implantadas en territorio dominicano (zonas francas industriales). En una de las regiones con mayor concentración de este tipo de industria, la mano de obra femenina es de un $81,5 \%$ con respecto a la masculina (Duarte, 1986).

La discriminación sexual dentro del mercado de trabajo se encontraba fundamentada jurídicamente hasta la fecha reciente de 1992 en la que se introduce el nuevo Código del Trabajo. Hasta entonces, las mujeres en la República Dominicana, al igual que los menores, en lo referido a la legislación laboral se encontraban dentro de un "estatuto especial». Este estatuto establecía diferencias discriminatorias con relación al acceso, horario y tipo de trabajo a realizar. Así, las mujeres se encontraban limitadas e inhabilitadas para ejercer los trabajos no apropiados a su sexo. El Código del Trabajo dominicano exigía que «toda mujer que pretenda realizar labores en empresas de cualquier clase acreditará su aptitud física para desempeñar el cargo de que se trate con una certificación médica» y que las mujeres «no podrán ser empleadas ni trabajar durante la noche» (Báez, 1987: 20).

La emigración fuera del país es una estrategia económica frecuentemente adoptada por los grupos domésticos en la República Dominicana de forma indistinta por hombres y mujeres. La participación de la mujer ha sido muy elevada y presenta porcentajes de representatividad similares a la masculina. Así, en EEUU, país donde hay un mayor número de población inmigrante dominicana, el porcentaje de mujeres con respecto a los hombres que han emigrado a EEUU oscila en torno al 49,8\% ${ }^{7}$. Las mujeres han emigrado junto con sus familiares hombres, dentro del modelo de familia nuclear de clase media en el que el hombre es el principal proveedor económico, pero también ellas han encabezado la emigración de sus hogares. A pesar de que ésta ha sido la tónica general, la utilización de esta estrategia por los hogares de la región suroeste se ha hecho cada vez más frecuente desde mediados de la década de los ochenta, siendo fundamentalmente mujeres las que salen de sus comunidades con destino a España. Las mujeres dentro de este flujo representan un $91 \%$ con respecto a los hombres.

6. Fenómeno por otro lado muy común en toda Latinoamérica (Buvinic y otros, 1983)

7. Según datos de elaboración propia a partir de los resultados de la encuesta ENDESA-1991. Este porcentaje, sin embargo, varía según fuentes y autores. 
Resumiendo, por lo que se refiere al contexto de origen de las mujeres inmigrantes dominicanas encontramos que éstas contribuyen a través de múltiples estrategias productivas a la reproducción de sus hogares. A pesar de ello, el valor de su trabajo, sea dentro del mercado de trabajo formal o informal, no es reconocido ni social ni económicamente. Esto genera situaciones de auténtica desesperación económica por mantener sus hogares, ya que a la situación descrita se unen además circunstancias como una tasa de fecundidad elevada ${ }^{8}$ y una ausencia de corresponsabilidad de los progenitores varones?

En cuanto a la sociedad marroquí, ésta prima la figura de la mujer como ama de casa, madre y esposa, y penaliza el descuido o abandono de este papel. En Marruecos está instituida una división de papeles en la cual a los hombres les corresponde ganar el sustento y a las mujeres, criar a los hijos. Incluso jurídicamente está regulado este reparto ${ }^{10}$. Ello está sancionado por el islam: «Dios creó de manera diferente a hombres y mujeres», «Dios dijo...», etc. ${ }^{11}$. Pero esta legitimación religiosa de la diferencia no ha de ocultar otra, que podría llamarse prelegitimación o justificación de tipo natural o biológico: «hombres y mujeres son diferentes». No es fácil saber dónde empieza la una y termina la otra.

En este contexto, el trabajo femenino exterior — salvo los casos de trabajo profesional y no siempre- es visto no como algo deseado y reivindicado, y que ayuda a conseguir autonomía, sino como una obligación y, en ocasiones, como una pesada carga. El papel de ama de casa es el más legitimado en Marruecos en el grupo de origen de las trabajadoras domésticas, en tanto que el papel de trabajadora no disfruta de esta legitimación. Ello tiene como consecuencia que ni el Estado ni la sociedad instituyen mecanismos que posibiliten que el trabajo revierta en recursos para la igualdad entre hombres y mujeres, o para un reparto más equitativo de las tareas domésticas en el interior de los hogares. Como marco de esta situación no puede dejar de señalarse la posición de las mujeres en Marruecos respecto a los derechos civiles. Desde la sociedad marroquí, definida como musulmana, se mantiene un discurso en el que aquélla — en su calidad de Umma o comunidad de creyentes - se erige en protectora de las mujeres, a las que considera eternas menores de edad y con per-

8. Según datos de la Encuesta Demográfica y de Salud de 1991, la tasa de fecundidad era de 2,8 referida a las mujeres urbanas y de 4,4 para las mujeres rurales.

9. Una de las leyes más incumplidas por los hombres en la República Dominicana es la Ley 2402 o Ley de la paternidad responsable, según se deduce del estudio realizado por CIPAF, 1985.

10. La Mudawana o Código de la Familia y el Estatuto personal así lo prescribe. Como ejemplo, baste el primer texto del Código:

Le mariage a pour but la vie dans la fidélité, la pureté et le désir de procréation par la fécondation, sur des bases stables et sour la direction du mari, d'un foyer permettant aux époux de faire face à leurs obligations réciproques dans la sécurité, la paix, l'affection et le respect mutuel.

11. La socióloga Fatima Mernissi cuenta una pequeña anécdota: en su calidad de feminista, es acusada por algunos colegas suyos de ir contra la Zurat, contra la tradición, contra el patrimonio, en el sentido de religioso-cultural (Mernissi, 1988). 
sonalidad jurídica dependiente siempre de un varón (que en las casadas es obligatoriamente el marido).

Los datos sobre la ubicación laboral de las mujeres en Marruecos y la manera en la cual se reflejan en las estadísticas no parecen escaparse de estas consideraciones. Hay tres cuestiones que tener en cuenta a la hora de evaluar estos datos. En primer lugar, existe una tendencia a la ocultación de las mujeres en censos, padrones y estudios. Simplemente no se registran. En segundo lugar, dado que el trabajo femenino es considerado como "ayuda» normalmente, aunque medie un salario, y considerando la situación del mercado de trabajo en Marruecos, en la que la mayoría de la producción se lleva a cabo de manera informal, la actividad de las mujeres está doblemente sumergida. Una tercera cuestión importante es la existencia del trabajo infantil. Este trabajo tampoco se declara como actividad laboral, sino que las niñas y niños en esta situación suelen agruparse en la rúbrica de «ayuda familiar» o «aprendices».

Existen diferencias importantes entre el campo y la ciudad en cuanto a la participación de las mujeres en los diferentes sectores económicos. En el campo, el porcentaje de mujeres en la población activa es del $42,9 \%$, en tanto que en la ciudad es del 25,6\% (CERED, 1990). El trabajo de las mujeres, según los autores, se caracteriza por una alta tasa de paro y de subempleo ${ }^{12}$. Este rasgo del subempleo parece acompañar la vida laboral de las mujeres, y puede decirse que muchas veces forma parte de la caracterización de la situación de las mujeres inmigrantes en España.

En el campo las mujeres se encargan sobre todo de cuidar el ganado. No hay que dejar escapar que esta actividad, que es fundamental en el mantenimiento del grupo doméstico, tradicionalmente se adjudica a la mano de obra teóricamente más débil: niños, viejos y mujeres. Simbólicamente, es una de las actividades más marginales.

Uno de los sectores de trabajo femenino más importantes en Marruecos es el servicio doméstico. Para las zonas rurales, sin embargo, no consta este sector, y en la ciudad el servicio doméstico apenas ocupa a un 20\% de la población activa femenina. No hay que concederle, en todo caso, mucho crédito a los datos sobre servicio doméstico en el campo, porque no existen instrumentos para medirlo. Y además, ¿qué es el servicio doméstico en Marruecos? ¿El hecho de que una chica viva con una familia que no es la suya y le den de comer es servicio doméstico?

En Marruecos, el servicio doméstico está reservado a las mujeres y a las niñas que pertenecen a grupos socioeconómicos con muy poco poder adquisitivo, y que no tienen más opción que trabajar. Esta obligación de trabajar las aleja de lo que ellas consideran el modelo para una mujer marroquí: el ocuparse a tiempo completo de su casa y su familia, modelo asociado a la ideología islámica. El servicio doméstico no es considerado por las propias trabajadoras

12. Los autores entienden por subempleo una ocupación de menos de 32 horas en medio urbano y de menos de 40 horas en medio rural que deja tiempo libre y la trabajadora busca otro empleo (CERED, 1990). 
un trabajo, que puede eventualmente dignificar a la persona que lo realiza, sino una carga pesada. Se espera abandonarlo en el momento del matrimonio. No se dispone de datos que relacionen el trabajo en el servicio doméstico con el estado civil. Sí existe este dato para el conjunto de los sectores económicos. En este sentido, no deja de ser interesante que la tasa de actividad es más del doble en las mujeres viudas o divorciadas $(31,2 \%)$ que en las casadas $(16,1 \%)$ y solteras (13,4\%), según datos de 1986 (CERED, 1992). Sin embargo, la tasa de actividad de las mujeres va descendiendo en las épocas en las que la crianza de los hijos requiere más atención (19-29 años), para volver a subir y alcanzar otro máximo en torno a los 40 años (CERED, op. cit: 61).

Hay grandes posibilidades de que el trabajo al que se está refiriendo el dato sea el servicio doméstico, trabajo asumido sobre todo por las mujeres, probablemente con cargas familiares, y que no tienen quien se ocupe de ellas. Sin embargo, hay que volver a considerar que una gran parte del trabajo femenino se lleva a cabo en Marruecos de modo sumergido, y que uno de los sectores con más alto índice de informalidad es el servicio doméstico, sobre todo el que es realizado por menores. El servicio doméstico en Marruecos trae consigo una serie de connotaciones peyorativas: relaciones de subordinación de la trabajadora respecto a los empleadores, bajísimos salarios, falta de garantías y seguro social, etc. Hay que decir que comparte algunos de estos rasgos con otros empleos, que también salen perjudicados de la ausencia absoluta en Marruecos de un Estado del bienestar. La cuestión es que en el servicio doméstico se ponen de manifiesto las grandes diferencias que existen en Marruecos entre los grupos sociales.

Los recursos generados por el servicio doméstico, dados los grupos sociales de pertenencia de las trabajadoras, suelen ir a parar al presupuesto familiar. No es habitual que esos recursos sean utilizados por las propias mujeres que los generan. En esta cuestión, sin embargo, hay una diferencia entre las mujeres que aún no tienen una familia, las solteras, y las que sí contribuyen al mantenimiento de su propia familia: esposo y/o prole. Otra cuestión importante es que las mujeres casadas necesitan la firma de su marido para poder ser parte de un contrato de trabajo. Las no casadas pueden hacerse cargo del contrato ellas solas. Esto es importante, pero sólo para una parte de la población que considera el trabajo femenino como algo deseable, y esta parte suele coincidir con las mujeres que tienen una profesión o que pueden acceder a un trabajo cualificado.

En el primer caso, el de las mujeres solteras, y a partir de la información de la que se dispone, el trabajo y la entrega de los recursos generados por ese trabajo a la familia es algo que forma parte de las funciones como hija o como hermana. Su posición, de hija y hermana, frente al padre y a los hermanos varones no cambia en la familia aunque trabaje. En este tipo de familias, las chicas no tienen gran capacidad de intervención en las decisiones familiares. Se trabaja para la familia, y ello fortalece las relaciones de subordinación, no al contrario. De hecho, sobre todo en lo que se refiere al trabajo infantil y adolescente, es la familia de la pequeña trabajadora quien arregla el salario con la 
familia empleadora. La chica a veces ni siquiera sabe lo que cobra, porque el salario es recogido directamente por su padre o por sus hermanos.

En el segundo caso, las mujeres que tienen sus propios hogares, puede establecerse aún otra clasificación: en el primer grupo se sitúan las mujeres no casadas con cargas familiares, que son probablemente las que copan las categorías de viuda y divorciada trabajadoras y después, como segundo grupo, las mujeres casadas. En ambos casos, el salario, como veremos más abajo, tampoco permite generalmente una negociación que mejore la posición de las mujeres en la familia o en el entorno, o que permita una redistribución equitativa del trabajo doméstico. Significa esto que sólo las mujeres que se consideran pertenecientes a familias muy pobres y en cierto punto, marginales, trabajarán en el servicio doméstico. En este sentido, muy pobre es una familia si permite que sus hijas vayan a vivir a casas de otros y trabajen para otros desde niñas. Desde este punto de vista, el trabajo femenino sería un criterio de estratificación social. Sin embargo, la discriminación de género tiene un importante papel, ya que es posible encontrar en la misma familia a niñas que trabajan en el servicio doméstico y que tienen hermanos que han llegado hasta la enseñanza secundaria.

No existe una tradición migratoria entre las mujeres marroquíes. La penalización de la movilidad femenina autónoma ha determinado que las mujeres sean siempre «las que se quedan» y los hombres, «los que se van». Hay, sin embargo, un dato interesante, y es que, si se toma la movilidad para la formación de un matrimonio, la mayoría de las mujeres marroquíes serían inmigrantes. Este movimiento es considerado como algo clave en la vida de una mujer: es el movimiento que se produce desde la casa de su padre - su primer tutor — hasta la casa de su marido — su segundo tutor ${ }^{13}$.

Las mujeres marroquíes no comienzan a emigrar hasta bien entrada la década de los años ochenta. España e Italia se introducen como destinos migratorios para estas mujeres, que comienzan a llegar simultáneamente a sus maridos, padres o compatriotas. Éste es un rasgo importante de la emigración hacia España, marcando una ruptura con respecto a movimientos migratorios anteriores. Respecto de Marruecos, el país de origen, porque es la primera vez que salen mujeres (vinculadas o no con hombres) para insertarse en el mercado de trabajo en destino; respecto al país de acogida, porque, al contrario que en la emigración hacia Europa del norte y central, las mujeres comienzan a emigrar desde casi el principio, a la vez que los hombres, sin tener que esperar a procesos de reagrupación familiar para asentarse en el país de inmigración (Ramírez, 1998).

Entre las mujeres marroquíes inmigrantes con las que se ha trabajado, la inmigración constituyó su primera experiencia laboral o prácticamente la primera. No es muy habitual encontrar mujeres que se dedicaran al trabajo en servicio doméstico antes de venir a España, excepto en casos aislados (Ramírez, 1998; Ramírez y López, 1999). Según TEIM (1992), apenas un 15\% de las

13. De hecho, y según un refrán popular marroquí, una mujer honrada sólo deberá salir dos veces de su casa: desde la casa de su padre hasta la casa de su marido, y desde la casa de su marido hasta el cementerio (citado por Lemrini, 1994) 
mujeres trabajaba en servicio doméstico antes de venir a España. Esto se debe probablemente a que las mujeres que vienen a España están en un estrato un poco más alto que las que se dedican al servicio doméstico en Marruecos. Como experiencia laboral anterior, hay lo que antes se ha denominado subempleo: pequeños trabajos que estas mujeres llevan a cabo, y por los cuales reciben recursos cuya suma no llega a constituir un salario.

\section{Mujeres dominicanas y marroquíes: diferencias en la percepción de su emigración laboral}

Una de las diferencias que se puede establecer entre las mujeres inmigrantes a las que nos referimos es su diferente papel en sus hogares con relación al grado de su contribución económica. Ello es un aspecto fundamental cuando se habla de mujeres inmigrantes trabajadoras cuyos familiares han quedado en origen. En ambos casos estas mujeres contribuyen de manera importante durante la inmigración al mantenimiento de sus hogares. Si en el caso de las mujeres dominicanas este aspecto supone una continuidad con el papel que venían desempeñando antes de salir, no es así en el caso de las mujeres marroquíes.

En la sociedad dominicana, como se ha comentado más arriba, el papel de las mujeres en la contribución económica al hogar es fundamental y este papel se seguirá manteniendo con su emigración. Hay que tener en cuenta, para analizar este tema, que un porcentaje importante de las mujeres que han emigrado, ha dejado a sus hijos en el país de origen a cargo de diferentes familiares, generalmente sus madres o hermanas. Los datos de una encuesta realizada a 210 mujeres dominicanas procedentes de la región suroeste ${ }^{14}$ son reveladores en este sentido: todas ellas envían dinero y un 70,5\% manda a sus familiares mensualmente más de 25.000 pesetas. Esta cantidad supone como mínimo asegurar el coste mensual de la canasta familiar básica, que según cifras oficiales del año en el que se realizó la encuesta (1995) estaba aproximadamente sobre esta cantidad. Incluso su papel como mantenedoras de sus hogares se ha visto reforzado con su emigración cuando los hombres — sobre todo sus esposos e hijos- abandonan sus trabajos una vez que las mujeres comienzan a mandar dinero de España. Como describía la situación un informante de la comunidad de origen de estas mujeres: «[...] generalmente la mujer ha sido la que ha tenido que emigrar porque sufre en carne viva la crisis que está sufriendo nuestro país y el hombre generalmente se queda esperando que la mujer comience a mandar dinero».

En la sociedad marroquí, como ya se examinó más arriba, el mantenimiento de la familia corresponde — incluso está ratificado por la Ley_ a los miembros varones. Primero el padre de la mujer y luego su esposo ${ }^{15}$. La emigración de las mujeres marroquíes no ha supuesto, en la mayor parte de los

14. Ver Gregorio Gil (1996).

15. De hecho, la falta de sostenimiento del hogar por parte del marido es una de las escasas razones por las cuales una mujer puede solicitar el divorcio según el Derecho malequí, por 
casos, un relevo de este papel. Sin embargo, ello no significa que no contribuyan al mantenimiento de hogar. De hecho esta contribución es un cambio fundamental.

El grado de presión económica que tienen las mujeres de ambos grupos condiciona su posición como inmigrantes. Y ello ocurre tanto en lo referido a las estrategias económicas que deben desplegar, como a la negociación de su posición de género en cuanto a la mayor o menor orientación de sus estrategias hacia su grupo doméstico o hacia ellas mismas. En el caso de las mujeres dominicanas se ha observado que para seguir cumpliendo con las responsabilidades hacia sus hogares, la mayor parte de sus estrategias económicas se dirigen a optimizar sus recursos para poder alcanzar este fin. Así, se ven obligadas a mantener su trabajo como domésticas internas, con el objeto de ahorrar costes de manutención en España y poder enviar periódicamente una parte importante del salario. Para las mujeres que sienten como una necesidad conseguir objetivos más personales, — como puedan ser: mejorar su formación, tener tiempo libre, dedicar parte de su ingreso a gastos propios, realizar viajes con más frecuencia a su país, etc.- la situación a la que se enfrentan hace muy difícil su logro reforzando su posición de género dentro de su hogar y en el mercado de trabajo. Por supuesto, a esto hay que añadir las limitaciones reales que el mercado de trabajo español impone a las mujeres inmigrantes.

Respecto a las mujeres marroquíes, y como ya se explicó en otro lugar (Ramírez, 1998), una manera de evitar la hachuma ${ }^{16}$ por ser inmigrante es asumiendo el mantenimiento de la familia. Esto les permite a las inmigrantes marroquíes ir ganando cuotas de poder y prestigio en su entorno. Sin embargo, no existe el grado de responsabilidad con la familia en origen que se encuentra entre las mujeres dominicanas. Si la familia que queda en Marruecos es la de orientación (padres, hermanos, abuelos), la inmigrante dejará de enviar remesas al contraer matrimonio, y este comportamiento estará legitimado. Aun en el caso de que permanezcan solteras y no dispongan de su propia familia, el imperativo del envío de dinero no será tan fuerte como para que condicione su posición dentro del mercado de trabajo. No es habitual, como sí lo es en el caso de la inmigración dominicana, que las mujeres marroquíes dejen al marido y a los hijos en origen. Esta segunda posibilidad puede existir, pero la responsabilidad parece reducirse al mantenimiento de los hijos, no al de toda la familia que cuida los hijos. Lo importante es que en el caso de la inmi-

el cual se rige Marruecos para la regulación de los contratos de matrimonio, divorcio, herencia y filiación, y que tiene su concreción en la Mudawana marroquí (V. Art. 53, cap. II, libro $2^{\circ}$ de la Mudawana).

16. Hachuma significa 'vergüenza, pudor'. Tener hachuma es tener vergüenza, y ello significa avergonzarse de hechos o situaciones que no son «normales» desde la posición que cada uno ocupa en la sociedad. En cuanto que la movilidad autónoma femenina y la inserción en el mercado de trabajo no son considerados atributos de mujeres, las inmigrantes, como otros transgresores de las normas, «compran» su decencia, su honor teóricamente perdidos. En el caso de las inmigrantes, ello se traduce enviando mucho dinero a la casa familiar. 
gración marroquí, la responsabilidad por el mantenimiento de la familia es compartida, no es la inmigrante la única responsable del grupo.

En suma, la inmigrante marroquí se hace cargo de la familia para poder conseguir algo extra (renegociación de su posición, prestigio, etc.), mientras que la inmigrante dominicana asume este papel como un objetivo prioritario en su emigración.

Es fundamental considerar aquí la diferente organización doméstica en función de las relaciones de parentesco y género. En el caso de la sociedad dominicana, las mujeres se apoyan fundamentalmente en sus redes familiares femeninas para repartir el trabajo doméstico; es habitual la residencia de tipo matrilocal, en el marco de la cual se establecen relaciones que tienen un papel fundamental, tanto en la cohesión de la familia como en su reproducción social. Esto influye claramente en el papel que las mujeres asumen dentro de sus hogares durante la inmigración, en el sentido de que son ellas las que de hecho llevan el peso fundamental de la reproducción de sus hogares, sean las que quedan en origen o las que emigran. En el caso marroquí, la norma es la patrilocalidad. En el pasado, ello respondía a una estructura social de tipo patrilineal. Actualmente, y si bien existe una consideración de la neolocalidad como lo deseable, es bastante común la patrilocalidad. Ello supone que es prioritaria la relación padres-hijo, en detrimento de la relación padres-hija. La hija pertenecerá siempre a otra familia, a la de su marido cuando contraiga matrimonio. Y todo ello supone que la hija siendo inmigrante - no será responsable de su familia de orientación. Aún así, y en una primera etapa, la inmigrante puede contribuir de manera importante a la mejora de las condiciones de vida de la familia, que muy probablemente revertirán en la mejora de vida de los hermanos varones y sus familias, que son los que permanecen unidos a los padres. De hecho, lo más probable es que las remesas enviadas lleguen a los hermanos de la inmigrante, aunque constituyan una unidad doméstica separada, y no lleguen a las hermanas en el mismo caso.

Otro aspecto que vamos a analizar es el referido a los cambios que pueda provocar la obtención de un salario mayor en la inmigración que el obtenido en el país de origen y ello con relación a si su distribución se orienta en mayor o menor medida hacia fines propios o hacia fines que tienen que ver con los «otros».

En el caso de las mujeres dominicanas, la obtención de un ingreso monetario mayor al que podían obtener en su país les permite destinar parte de su salario a fines propios, no directamente relacionados con el mantenimiento de sus hogares, como por ejemplo tomar una bebida con amigos y amigas fuera de casa, comprarse ropa o joyas, viajar, etc. Anteriormente, esto era prácticamente imposible por la escasez económica bajo la que se dirimía la vida en sus hogares. Sin embargo, se ha observado que el gasto en bienes más personales siempre se hace con el sobrante que pueda quedar una vez cubiertas las necesidades de sus familiares. Como comentaba una mujer inmigrante dominicana:

Por ejemplo, yo me pude comprar unas cuantas prendas, porque soy muy vanidosa, sí es verdad, estas prendas que yo me compré que se entienda que esto fue de mis horas extras, porque lo mío estaba como te digo... ¿ ¿Di que yo ir a una 
joyería y dar 30.000 pesetas por un... necesitándolo aquí mis hijos de comida o de ropa y cosas más necesarias?

Se podría concluir que sigue dominando su responsabilidad como madres, que les hace poner en primer lugar los intereses de su grupo familiar, antes que los suyos propios.

No es el caso de las mujeres marroquíes, ya que no parece existir una diferencia apreciable en la proporción de gasto que en la inmigración dedican a ellas mismas y la que dedicaban a los mismos fines estando en Marruecos.

Un último aspecto que queremos abordar es el de las repercusiones que el trabajo dentro del servicio doméstico y de la prostitución tiene para estas mujeres. Ambos trabajos son generizados y típicamente femeninos, no suponiendo su desempeño una ruptura. En el caso del servicio doméstico, es una continuidad con el rol de esposa y madre; en el de la prostitución, se adopta el papel de proveedora de otra necesidad masculina, el sexo. Ninguno de los dos roles les son desconocidos a estas mujeres. A estos empleos se ven forzadas a recurrir en la mayoría de los casos, y ello tanto en su sociedad de origen como en España, ante la desposesión de otros recursos que le garanticen su bienestar y el de los suyos. En sus sociedades de origen, con diferencias de grado, ambos trabajos tienen en común la carga de desprestigio social que llevan añadida. Tanto en Marruecos como en la República Dominicana el servicio doméstico tiene una connotación de clase que añade a los que ejercen este trabajo un aspecto más de la subordinación de unos grupos respecto a otros; en la República Dominicana además se añaden connotaciones de «raza», dado que en este país la pobreza está muy unida con el origen africano o haitiano ${ }^{17}$.

Por lo que se refiere a lo que está significando para las mujeres marroquíes y dominicanas su inserción en el servicio doméstico madrileño, encontramos un elemento común y otro diferencial. Para ambas mujeres trabajar fuera del hogar se constituye en un valor aunque sea interpretado de manera distinta. Sin embargo, para las mujeres marroquíes, la connotación de clase desaparece, en tanto que no es así para las mujeres dominicanas, como se explica más adelante.

Para las mujeres marroquíes el servicio doméstico en la inmigración posibilita la «dignificación de la mujer» como trabajadora. Aparece el trabajo exterior como un valor y no tanto como una carga. El concepto de trabajo exterior se transforma y se termina integrando como actividad propia sin que caiga en contradicción con una vida familiar normal, sobre todo en lo que se refiere al servicio doméstico externo. En definitiva, la actividad laboral comienza a tener un valor propio para las mujeres y deja de ser algo relacionado con la carga, obligación o posición de clase. Para las mujeres dominicanas, con la inmigración no aparece la valoración del trabajo fuera del hogar; para éstas, el trabajo digno siempre ha sido un valor - tener un negocio propio, un empleo más

17. Existe un término en la República Dominicana con connotaciones negativas para designar a la mujer que trabaja como doméstica en las casas de otros y cuyo color de piel es negro, dicho término es chopa. 
o menos estable, etc. No lo es, sin embargo, trabajar en el servicio doméstico. Aunque hay que decir que, en España, el salario que reciben trabajando como domésticas compensa la carga negativa a la que se tiene asociado el servicio doméstico en la República Dominicana. Allí no hay establecido un salario sino un intercambio de bienes; si hay sueldo, es ínfimo. Ahora su trabajo en España es digno porque permite un ascenso socioeconómico considerable con respecto a su sociedad de origen. Para las mujeres cuya actividad principal se centraba en su hogar, la manera de dignificarlo es considerándolo como una continuación de sus tareas domésticas pero ahora remuneradas. Como algunas mujeres comentan: «me pagan por hacer lo mismo que en mi país». Esta valoración del trabajo doméstico, sin embargo, no es llevada a cabo por aquellas mujeres a las que la emigración les ha supuesto renunciar al ejercicio de profesiones de mayor prestigio (médicas, enfermeras, maestras, etc.). Éstas últimas manifiestan de manera constante la carga de tener que dedicar prácticamente todo su tiempo al desempeño del trabajo en el servicio doméstico, renunciando a la realización de otras actividades.

En definitiva, aunque para las marroquíes trabajar en el servicio doméstico sea parte de la novedad de emigrar en todos los sentidos y les es más fácil redefinirlo en función de sus nuevas circunstancias, no es así para la mayoría de las mujeres dominicanas. Aun considerando que para ambas trabajar fuera de sus hogares constituye un aspecto muy valorado que les permite en mayor medida decidir sobre sus vidas, para las mujeres dominicanas lo es con relación al ingreso que proporciona y no tanto al hecho de trabajar fuera del hogar. Este ingreso les permite estar orgullosas por poder mantener a sus hijos y tener una mayor independencia monetaria de sus esposos. Así lo destacaba una mujer entrevistada: «Sí, yo nunca hubiera trabajado y ahí sé lo que era trabajar — se refiere a España - era un regocijo porque una puede darse a sus hijos y a su familia». También otra mujer dominicana al ser preguntada por los cambios experimentados a raíz de su emigración señaló: «Igual, lo único que aquí —en la República Dominicana — se trabajaba y ganaba menos y allí — se refiere a España- trabajo y gano más».

Aun teniendo en cuenta lo anterior entre los dos grupos, sin embargo, hay que decir que el modelo de mujer, madre y esposa, de clase media, mantenida por su marido, es una aspiración sostenida de modo más o menos ficticio por ambos grupos. Para las marroquíes, la explicitación de esta aspiración pone de manifiesto, una vez más, el deseo de ser consideradas como «chicas normales y buenas», que lo que quieren es ser amas de casa, que no lo son por necesidad, y que esa transgresión que supone el trabajo exterior se vive como algo transitorio. Para las mujeres dominicanas este deseo descansa en la casi exclusiva valoración social de las mujeres a partir de su maternidad y de su relación conyugal con un hombre. Añadiéndose, ante las condiciones de pobreza que rodean al trabajo de las mujeres de los sectores más desfavorecidos, la idea de que la existencia de un hombre en el hogar puede contribuir a la mejora económica de éste.

En el caso de las marroquíes, el trabajo en el servicio doméstico externo en la inmigración parece haberse desposeído de todos los elementos negativos de 
diferencia de clase, que eran claves en la relación laboral entre empleador y empleada en Marruecos. Sin embargo, no ocurre así con el servicio doméstico interno, que sí que suele ser contemplado como algo negativo, sobre todo debido a la residencia continuada en casa de los empleadores y a la disponibilidad absoluta que tiene la empleada para desempeñar su trabajo a cualquier hora del día o la noche. Aunque hay otros trabajos mejor considerados, el servicio doméstico externo parece haberse revestido, en la inmigración, de cierta honorabilidad de la que carecía en origen.

En cuanto a las inmigrantes dominicanas, éstas experimentan un descenso en su estatus, respecto a la situación de origen, al integrarse en el servicio doméstico, sobre todo en la modalidad de internamiento. Y ello es así tanto para las que poseían un empleo cualificado en su país de origen, como para las que su actividad principal se centraba en el trabajo dentro de su hogar. Las mujeres dominicanas experimentan como una desventaja tener que trabajar como «domésticas», a pesar de la valoración positiva que hacen de los ingresos que reciben. Un dato interesante al respecto es que un 34,2\% de las mujeres que fueron encuestadas ante la pregunta "¿qué hace en España que no hacía en su país?». Su respuesta fue: "trabajar en el servicio doméstico»" ${ }^{18}$. Ello nos indica la vivencia negativa del cambio en cuanto que en su país era algo de lo que estaban alejadas y aquí se ven «obligadas» a hacer.

Por lo que se refiere al trabajo sexual, no tenemos datos estadísticos fiables de las mujeres dominicanas y marroquíes que lo desempeñan en cualquiera de sus modalidades. Pero sobre la base de algunas entrevistas realizadas a mujeres marroquíes y dominicanas que trabajan en este sector, podemos decir que la vida de estas mujeres cambia en mayor medida que las que se dedican al servicio doméstico, al menos por dos razones. Por un lado, porque trabajar en la prostitución supone la realización de actividades nuevas para la mayoría, ya que lo habitual es que no se dedicasen a ello en su país de origen. Por otro lado, porque este trabajo se desarrolla en un medio desconocido y más hostil en principio que el hogar, donde al fin y al cabo siguen las mujeres que trabajan como domésticas.

Tanto en el caso de las mujeres marroquíes como dominicanas hemos observado dos recorridos diferentes para llegar a la prostitución. Por un lado, algunas mujeres que han trabajado primero en el servicio doméstico deciden buscar un trabajo sexual, casi siempre como una estrategia de aumentar los ingresos y para poder tener una vida propia a la que no tienen acceso cuando trabajan como internas en una casa. Por otro lado, existen redes de prostitución tejidas desde los países de origen que no dejan muchas alternativas a las mujeres que viajan al amparo de éstas.

Con respecto a cómo se valora en Marruecos y en la República Dominicana a las mujeres inmigrantes que se insertan en el mercado del sexo, no parece que se las excluya o margine por el hecho de desempeñar este tipo de trabajo.

18. El porcentaje más alto —un 42,2\%—, por encima del señalado, contestó «tener mayor libertad para salir y hacer cosas» (Gregorio, 1998: 251). 
En la República Dominicana las «mariposas nocturnas» o «mujeres de la calle» 19 han pasado a recibir la denominación de "bailarinas», cuando trabajan dentro del mercado del sexo fuera de su país. Término, éste último, que simbólicamente supone una cierta profesionalización de su trabajo. Un factor que contribuye a la aceptación de estas mujeres es que los ingresos que perciben suelen ser mayores que en su país de origen y que las remesas que envían son más voluminosas que las de otras inmigrantes que desempeñan otros empleos. Para todas estas mujeres la aceptación por parte de su comunidad de origen se dirime en términos de si envían o no envían dinero a los familiares que se han quedado allí. Unas y otras salen de países en los cuales la situación de subordinación de las mujeres hace del cuerpo femenino un recurso con valor de cambio. En la República Dominicana las mujeres se ven obligadas a utilizar su cuerpo ante la privación de otros recursos, como se ve en el discurso de una mujer dominicana de avanzada edad, que transcribo a continuación: «Ese — señalándose el pubis - es el conuco ${ }^{20}$ que a una le han dado, porque si usted es joven y Dios le ha puesto eso ahí, eso le da jabón, ropa, zapatos... Pero aquí hay muchachitas que los dan por ná». En el caso marroquí, la distancia entre hombres y mujeres hace difícilmente concebible el sexo más allá de una estrategia de intercambio, que utilizan sobre todo las mujeres. El encuentro sexual como algo enriquecedor no es habitual. Puede ser que estas características condicionen un paso menos traumático desde un sector como el servicio doméstico a la prostitución.

\section{Conclusiones}

A partir de las diferentes situaciones inmigratorias analizadas podemos concluir que la emigración de estas mujeres está provocando cambios significativos en sus vidas. Aspectos como el desempeño laboral fuera del hogar, la obtención de un mayor volumen de ingresos que en sus países de origen, la ausencia del control familiar o la posibilidad de tomar más decisiones con relación al gasto de sus ingresos económicos constituyen nuevas formas de relacionarse con ellas mismas y dentro de su familia y de su entorno social, dividido ahora entre sus comunidades de origen y Madrid.

Como inmigrantes, las mujeres marroquíes y dominicanas comparten estos cambios, pero su posición ante éstos se ve afectada por una serie de factores, que hemos intentado analizar y que tienen que ver con la construcción específica de género en cada una de las sociedades de origen, de las que siguen siendo parte.

Con nuestra contribución hemos pretendido mostrar la complejidad que implica el análisis de los procesos de cambio en las relaciones de género, que en apariencia podrían parecer iguales para todas las mujeres inmigrantes.

19. Términos empleados en la República Dominicana para referirse a las mujeres que se dedican a la prostitución.

20. Parcela de tierra. 


\section{Bibliografía}

BÁEZ, Clara (1987). Las mujeres como beneficiarias de servicios financiados por recursos asignados en el presupuesto nacional de la República Dominicana. República Dominicana, Documento presentado a la CEPAL (inédito).

Buvinic, Mayra; Lycette, Margaret A.; Mc Greevey, Willian P. (eds.) (1983). Women and Poverty in the Third World. Baltimore y Londres: Foreign Policy Association, The John Hopkins University Press.

CELADE (1988). República Dominicana: población y desarrollo 1950-1985. Costa Rica: IEPD, CELADE, FNUAP, ACDI.

CERED. Direction de la Statistique (1990). Ménages. Variables socio-démographiques. Rabat.

- (1992). Femme et developpement au Maroc. Rabat.

CIPAF (1985). Paternidad responsable, un estudio de la Ley 2402. Santo Domingo: CIPAF.

DUARTE, Isis (1986). Trabajadores urbanos. Ensayos sobre la fuerza laboral en República Dominicana. Santo Domingo: Editora Universitaria. UASD.

GREGORIO GIL, Carmen (1996a). Sistemas de género y migración internacional: La emigración dominicana en la Comunidad de Madrid. Tesis Doctoral. Departamento de Sociología y Antropología Social. Universidad Autónoma de Madrid (microfichas).

- (1996b). "El proceso de integración de las mujeres inmigrantes». En AGUADO, $\mathrm{M}^{\mathrm{a}}$ José. Las mujeres inmigrantes y su integración social. Madrid: Ministerio de Asuntos Sociales. Instituto de la Mujer.

- (1998a). La migración femenina y su impacto en las relaciones de género. Madrid: Narcea de ediciones.

- (1998b). «Mujeres inmigrantes: procesos de inclusión y exclusión» V Congreso de Intervención social. Colegio Oficial de Psicólogos de Madrid. Edición en CDRom.

LEMRINI, Amina (1994). "Limage de la femme à travers le discours scolaire». En BARKALlil y otros. Femme et éducation. Blocages et impacts. Casablanca: Le Fennec, p. 55-83.

Mernissi, Fatima (1991). Chahrazad n'est pas marocaine. Casablanca: Le Fennec. Millán, Celia (1992). «Diagnóstico socioeconómico de las mujeres del Sur». Informe presentado a PROMUS. Santo Domingo (inédito).

MinisTĖRE DE LA JUSTICE (1993). Code du statut personnel et des sucessions (Mudawana). Casablanca: Institut National d'Études Judiciaires.

NAROTZKY, Susana (1988). Trabajar en familia. Mujeres, hogares y talleres. Valencia: Edicions Alfons el Magnànim.

PASTORA, Isabel (1985). «Mujer rural y vida cotidiana. Trabajo productivo/reproductivo». Seminario diagnóstico sobre la mujer rural dominicana. CIPAF, UNIFEM, Santo Domingo (inédito).

Pou, Francis y otros (1987). La mujer rural dominicana. Santo Domingo: CIPAF. RAMírEZ, Ángeles (1998). Migraciones, género e Islam. Mujeres marroquíes en España. Madrid: Agencia Española de Cooperación Internacional.

RAMírEZ, Ángeles; LÓPEZ GARCÍA, Bernabé (1999). «Aixa y los lobos: historia de una inmigrante». Cuadernos de África y América Latina. Barcelona (en prensa).

TEIM (1992). Encuesta a inmigrantes marroquies. Sin publicar.

VAldÉs, Teresa; Gomariz, Enrique (1993). «República Dominicana». En Mujeres Latinoamericanas en cifras. Madrid: Instituto de la Mujer-FLACSO. 